INRA Prod. Anim., 2011, 24 (4), 297-306

\section{Contraintes et opportunités pour l'amélioration génétique des ruminants}

E. VERRIER ${ }^{1,2}$, M. SAINT-DIZIER 1,3,4

1 AgroParisTech, Génétique, Elevage et Reproduction, 16 rue Claude Bernard,

F-75231 Paris, France

2 INRA, UMR1313 Génétique Animale et Biologie Intégrative, F-78350 Jouy-en-Josas, France

${ }^{3}$ INRA, UMR1198 Biologie du Développement et Reproduction, F-78350 Jouy-en-Josas, France

${ }^{4}$ ENVA, Biologie du Développement et Reproduction, 7 avenue du Général de Gaulle, F-94704 Maisons-Alfort, France

Courriel : etienne.verrier@agroparistech.fr

Espèces domestiques d'importance majeure en Europe, les ruminants se caractérisent par une grande diversité d'utilisations, de races, de systèmes d'élevage, de territoires exploités et de filières de produits. En ce qui concerne leur reproduction, ils se distinguent de beaucoup d'autres espèces animales par une faible fécondité des femelles et la lenteur du renouvellement des générations. Quelles sont les conséquences de ces caractéristiques sur le développement de programmes d'amélioration génétique, leur organisation, leur efficacité ? C'est à ces quelques questions que le présent article tente d'apporter des éléments de réponse.
L'amélioration génétique des anisitue à l'amont des filières de production animale. Les acteurs de ce secteur ont pour mission la fourniture des types génétiques les mieux adaptés aux conditions de milieu physique, économique et social dans lesquels ils seront exploités tout au long de chaque filière, du producteur au consommateur. Cette activité se développe à l'échelle de populations animales dans leur ensemble (races, lignées, souches...), dans le cadre de programmes très structurés qui comprennent toutes les opérations conduisant au choix raisonné des reproducteurs et à leur utilisation. Les différentes étapes de ces programmes sont les suivantes : $i$ ) la définition des objectifs de sélection, ii) la collecte des informations nécessaires, iii) l'évaluation génétique des animaux, $i v)$ le choix des reproducteurs parmi des candidats issus d'accouplements raisonnés et $v$ ) l'utilisation des reproducteurs. La manière dont ces étapes se déroulent, les méthodes mises en œuvre et l'organisation correspondante dépendent largement de l'espèce et de la filière considérées. Dans le présent article, nous nous intéresserons aux trois espèces de ruminants domestiques maux est un secteur d'activité qui se majeures en Europe, à savoir les bovins, les ovins et les caprins (pour une approche similaire concernant les chevaux, voir Verrier 2011). Nous indiquerons en quoi certains paramètres biologiques de ces espèces et certaines caractéristiques des filières où elles sont exploitées ont des conséquences sur les pratiques de sélection et leur efficacité, en suivant la chronologie des étapes rappelées ci-dessus. Auparavant, nous donnerons un aperçu des effectifs et de la localisation des cheptels concernés et de l'organisation des éleveurs pour l'amélioration génétique.

\section{1 / Le cheptel en France et en Europe, l'organisation collective des éleveurs}

\section{1 / Taille et localisation des cheptels}

A l'échelle mondiale, l'Union Européenne occupe une position de premier plan pour les productions issues de l'élevage des ruminants, tout particulièrement celle du lait (tableau 1). Le cheptel est inégalement réparti entre les différents pays : selon l'espèce, les
Tableau 1. Principales productions issues de l'élevage de ruminants au sein de I'Union Européenne (UE).

\begin{tabular}{|l|c|c|c|c|c|}
\hline & $\begin{array}{c}\text { Lait de } \\
\text { vache }\end{array}$ & $\begin{array}{c}\text { Lait de } \\
\text { brebis }\end{array}$ & $\begin{array}{c}\text { Lait de } \\
\text { chèvre }\end{array}$ & $\begin{array}{c}\text { Viande } \\
\text { bovine }\end{array}$ & $\begin{array}{c}\text { Viande } \\
\text { ovine }\end{array}$ \\
\hline $\begin{array}{l}\text { Production UE } \\
\text { (millions de } \\
\text { tonnes) }\end{array}$ & 147,3 & 3,0 & 1,8 & 7,9 & 0,9 \\
\hline $\begin{array}{l}\text { Rang de l'UE } \\
\text { dans le monde }\end{array}$ & $1^{\text {er }}$ & $1^{\text {er }}$ & $3^{\text {ème }}$ & $3^{\text {ème }}$ & $2^{\text {ème }}$ \\
\hline
\end{tabular}

Source : FAO-Stat, chiffres de 2009. 
Tableau 2. Effectifs des différentes espèces de ruminants au sein de l'Union Européenne (UE) et parts respectives du cheptel détenu dans les principaux pays.

\begin{tabular}{|l|c|c|c|c|}
\hline & $\begin{array}{c}\text { Vaches } \\
\text { laitières }\end{array}$ & $\begin{array}{c}\text { Vaches } \\
\text { allaitantes }\end{array}$ & Brebis & Caprins \\
\hline $\begin{array}{l}\text { Effectif total UE } \\
\text { (millions) }\end{array}$ & 23,7 & 12,3 & 67 & 13 \\
\hline dont (\%) & 17 & 6 & - & - \\
Allemagne & 4 & 17 & 22 & 22 \\
Espagne & 16 & 33 & 9 & 10 \\
France & - & - & 10 & 36 \\
Grèce & 5 & 9 & 4 & - \\
Irlande & 8 & 3 & 11 & 7 \\
Italie & 11 & - & - & - \\
Pologne & - & - & 3 & 4 \\
Portugal & 7 & - & - & - \\
Pays-Bas & - & - & 9 & 8 \\
Roumanie & 8 & 13 & 21 & - \\
Royaume-Uni & 24 & 19 & 11 & 13 \\
Autres & & & & \\
\hline
\end{tabular}

Source : FranceAgriMer et FAO-Stat, chiffres de 2009.
Ouest. L'élevage des petits ruminants se situe principalement dans la moitié Sud du territoire. Un quart des brebis est élevé dans la région Midi-Pyrénées et plus d'un tiers des chèvres en PoitouCharentes.

Le cheptel bovin et le cheptel ovin allaitant sont détenus par un grand nombre d'éleveurs (de plusieurs dizaines de milliers à plus d'une centaine de milliers), avec un nombre modéré de femelles reproductrices chacun (tableau 3). En revanche, les cheptels laitiers ovin et caprin sont, pour l'essentiel, détenus par un petit nombre d'éleveurs avec de grands troupeaux. Quelle que soit l'espèce, l'évolution de ces dernières décennies a consisté en un agrandissement des structures: par exemple, la taille moyenne des élevages de vaches laitières a été multipliée par six sur les trente dernières années, conduisant à d'importantes modifications de la conduite des animaux et des besoins des éleveurs (Verrier et al 2010). deux à quatre pays possédant les plus importants cheptels cumulent plus de la moitié des effectifs totaux (tableau 2). La France détient le premier cheptel de bovins allaitants de l'Union Européenne, le deuxième de bovins laitiers, le troisième de caprins et le quatrième d'ovins.

La répartition de ces cheptels en France montre des disparités (figure 1). Le cheptel bovin est présent sur quasiment tout le territoire, avec une prédominance des bovins laitiers au Nord et des bovins allaitants au Sud. Le cheptel laitier est relativement plus «concentré») que le cheptel allaitant: un quart environ des vaches laitières est élevé en Bretagne et la moitié dans le Grand
Tableau 3. Nombre de femelles reproductrices et nombre d'élevages de ruminants en France.

\begin{tabular}{|l|c|c|c|c|c|}
\hline & $\begin{array}{c}\text { Bovins } \\
\text { laitiers }\end{array}$ & $\begin{array}{c}\text { Bovins } \\
\text { allaitants }\end{array}$ & $\begin{array}{c}\text { Ovins } \\
\text { laitiers }\end{array}$ & $\begin{array}{c}\text { Ovins } \\
\text { allaitants }\end{array}$ & Chèvres \\
\hline Nb. total de femelles (x 1000) & 3700 & 4150 & 1623 & 4146 & 888 \\
\hline $\begin{array}{l}\text { Nb. total d'élevages } \\
(\times 1000)\end{array}$ & 82 & 118 & 5 & 52 & $5^{*}$ \\
\hline $\begin{array}{l}\text { Nb. moyen de femelles } \\
\text { par élevage }\end{array}$ & 45 & 35 & 334 & 79 & $143^{*}$ \\
\hline
\end{tabular}

Source : Institut de l'Elevage, chiffres de 2009.

* Elevages de plus de 10 chèvres uniquement.

Figure 1. Principales régions françaises d'élevage (a) de bovins et (b) de petits ruminants.
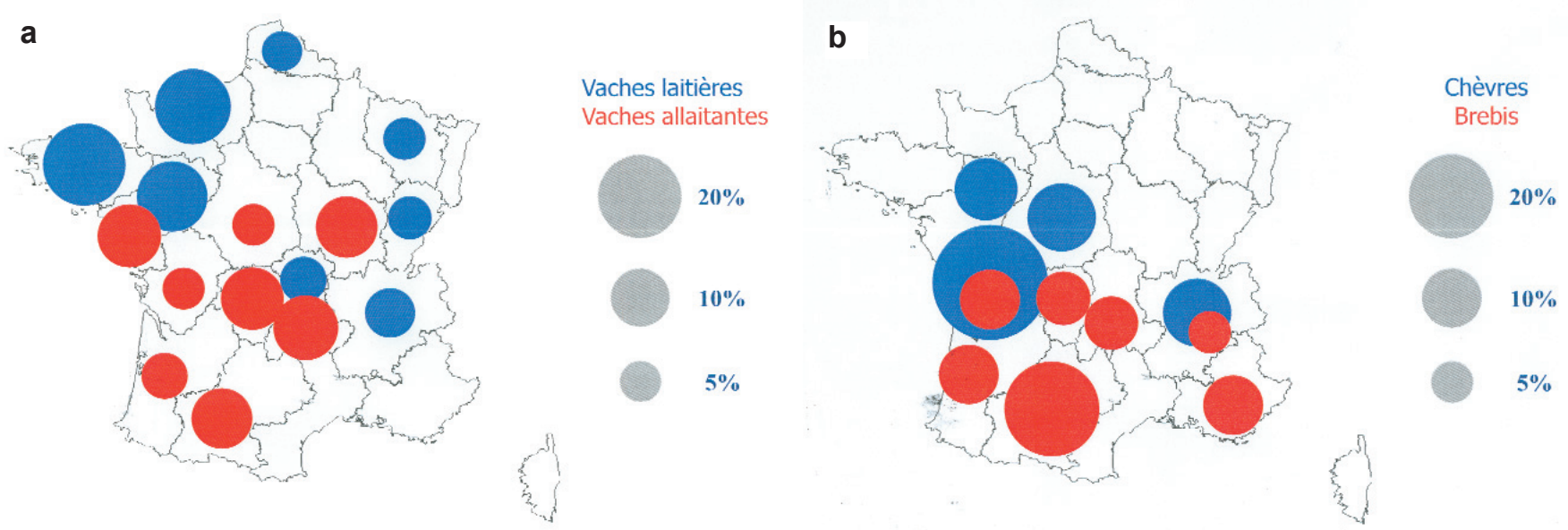

Source : Institut de l'Elevage-GEB (chiffres de 2010, sauf pour les chèvres, 2009). 
Photo 1. Vaches laitières Valdotaines en alpage (Valgrisenche, Italie).

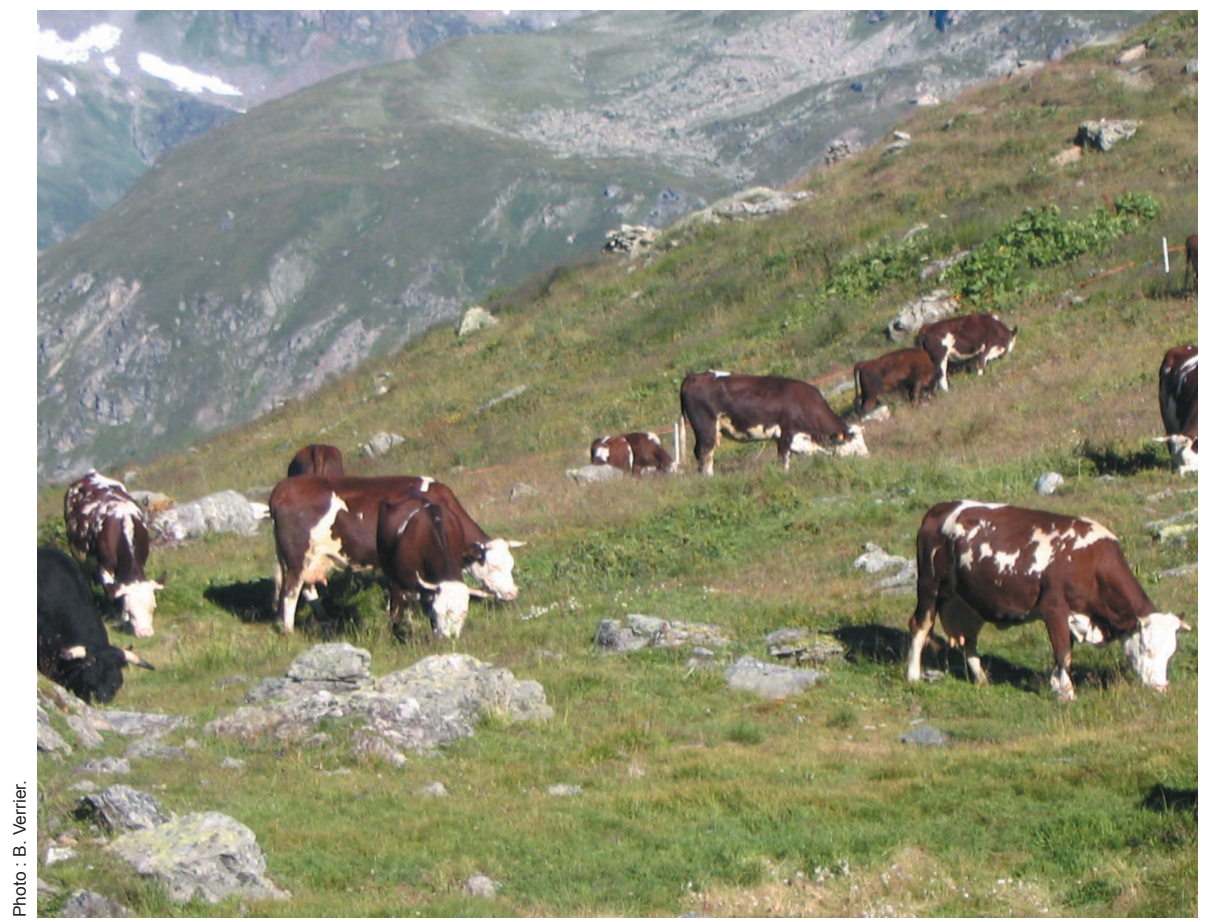

\section{2 / L'organisation de la sélec- tion}

La sélection consistant par essence en un tri des animaux et au repérage de ceux destinés à la reproduction du fait de leur valeur génétique, une des conditions de son efficacité est de pouvoir travailler sur des effectifs importants. Or les ruminants, même ceux qualifiés de «petits», représentent des coûts d'entretien importants, si bien qu'il est impossible pour une même personne morale d'investir dans des cheptels suffisamment nombreux pour garantir l'efficacité des programmes. Par ailleurs, ces espèces se reproduisant lentement (voir plus loin), le retour sur investissement serait beaucoup trop lointain pour un investisseur privé. L'introduction de la génomique dans les programmes de sélection permet certes d'amoindrir ces contraintes mais cellesci demeurent fortes chez les ruminants. Ainsi, les programmes de sélection des ruminants sont mis en place grâce à une mutualisation des moyens entre les éleveurs, qui sont à la fois producteurs et sélectionneurs (ou contributeurs aux programmes). La sélection est donc organisée de façon collective, les éleveurs étant impliqués dans les décisions de sélection. En France, les programmes sont conduits par des entreprises de sélection, sous forme de coopératives, d'unions de coopératives ou de groupements, et des organismes de sélection, sous forme associative.

La sélection collective des ruminants faisant intervenir de très nombreux opérateurs, elle fait l'objet de nombreux accords interprofessionnels et elle est fortement réglementée, tant à l'échelle nationale qu'à l'échelle internationale. En France, le cadre réglementaire a longtemps été celui de la Loi sur l'Elevage de 1966. Depuis la Loi d'Orientation Agricole (LOA) de 2006, la législation est nettement moins contraignante (hormis dans le domaine sanitaire) et l'interprofession est rassemblée au sein de France Génétique Elevage (voir par exemple, AgroParisTech-CSAGAD et Institut de l'Elevage 2006).

\section{2 / Populations, reproduc- teurs et paramètres de la reproduction}

\section{1 / Importance de la notion de race et de la valeur du géniteur}

L'amélioration génétique se conçoit et se pratique à l'échelle d'une population, c'est-à-dire d'un ensemble d'animaux de la même espèce qui ont la possibilité (au sens probabiliste du terme) de se reproduire ensemble. Pour les espèces de ruminants, une population est désignée par le terme de race, dont le code rural, dans son article D.653-9, donne la définition suivante : «un ensemble d'animaux qui a suffisamment de points en commun pour pouvoir être considéré comme homogène par un ou plusieurs groupes d'éleveurs qui sont d'accord sur l'organisation du renouvellement des reproducteurs et des échanges induits, y compris au niveau inter- national». La race a donc à la fois une acception biologique (points en commun) et une acception sociale (groupe d'éleveurs), le poids relatif de la seconde s'étant accru ces dernières décennies, tant dans l'approche scientifique que dans l'usage et la réglementation. En élevage des ruminants, la race est beaucoup plus qu'une notion, c'est une réalité (Lerner et Donald 1966, Denis 1982). L'amélioration génétique s'opère ainsi au sein de chaque race séparément, ce qui n'exclut pas les échanges de gènes entre races (cf. $\S 6.2)$.

Les espèces de ruminants se caractérisent par ailleurs par des liens encore forts entre les éleveurs et leurs animaux. C'est sans doute plus net avec les bovins qu'avec les petits ruminants (et plus encore avec ces autres grands herbivores domestiques que sont les chevaux). Compte tenu de ces liens et de la taille modérée des troupeaux de bovins, les éleveurs sont généralement attachés à l'identité des géniteurs utilisés et à la connaissance de leur valeur génétique individuelle, notamment celle des mâles. Les prix de vente spéculatifs de certains jeunes taureaux de races allaitantes ne sont qu'une illustration de ce phénomène. Compte tenu de tailles de troupeaux plus importantes, surtout en système laitier (tableau 3), les éleveurs de petits ruminants s'accommodent mieux d'une approche à partir de moyennes et non pas au cas par cas. Mais, connaître le type génétique des parents de leurs animaux sans en connaître les caractéristiques individuelles, ou au minimum, moyennes ne leur suffit pas (contrairement aux éleveurs de volailles, par exemple). Là encore, la sélection génomique, qui s'accompagne d'une utilisation en lots des jeunes taureaux, tend à faire évoluer les exigences des éleveurs de bovins laitiers dans ce domaine.

\section{2 / Importance des paramètres de la reproduction}

Dans la mesure où la sélection repose sur le renouvellement progressif d'anciennes générations de reproducteurs par de plus jeunes, sa mise en œuvre dépend étroitement des paramètres de la reproduction (tableau 4). La première mise à la reproduction des femelles est conditionnée par l'apparition de la puberté (6-7 mois chez les ovins et caprins, 10-18 mois chez les bovins) et la croissance des jeunes reproductrices, qui doit être suffisamment avancée pour mener à bien une gestation. Sous nos climats tempérés, la saison est une contrainte supplémentaire chez les petits ruminants : leur activité sexuelle débute en été (lorsque la durée du jour diminue) et se termine en hiver, avec 
Tableau 4. Principaux paramètres de la reproduction selon l'espèce et la filière.

FIV = Fécondation In Vitro $; \mathrm{IA}=$ Insémination Artificielle $; \mathrm{MN}=$ Monte Naturelle $;$ OPU = Ovum Pick-Up ; TE = Transplantation Embryonnaire ; $x=$ plusieurs.

a) Femelles

\begin{tabular}{|c|c|c|c|c|c|}
\hline & $\begin{array}{l}\text { Bovins } \\
\text { laitiers }\end{array}$ & $\begin{array}{l}\text { Bovins } \\
\text { allaitants }\end{array}$ & $\begin{array}{l}\text { Ovins } \\
\text { laitiers }\end{array}$ & $\begin{array}{l}\text { Ovins } \\
\text { allaitants }\end{array}$ & Chèvres \\
\hline Saisonnalité & non & non & oui & oui & oui \\
\hline Date $1^{\text {ère }}$ mise bas (ans) & $2-3$ & $2-3$ & $1-2$ & $1-2$ & $1-2$ \\
\hline $\mathrm{Nb}$. de mises bas par an & 1 & 1 & 1 & $1-1,5$ & 1 \\
\hline $\mathrm{Nb}$. de jeunes par mise bas & 1 & 1 & $1-3$ & $1-3$ & $1-3$ \\
\hline Femelles inséminées $(\%)$ * & $82 \%$ & $13 \%$ & $44 \%$ & $4 \%$ & $9 \%$ \\
\hline Maîtrise des cycles & \multicolumn{2}{|c|}{ Synchro. / Superovulation } & \multicolumn{3}{|c|}{ Synchronisation / Désaisonnement } \\
\hline Biotechnologies de l'embryon & \multicolumn{2}{|c|}{ TE/OPU/FIV } & & & \\
\hline $\begin{array}{l}\mathrm{Nb} \text {. total de descendants } \\
\text { sans biotechnologies }\end{array}$ & $3-4$ & $4-8$ & $6-12$ & $6-12$ & $8-12$ \\
\hline $\begin{array}{l}\text { Nb. total de descendants } \\
\text { avec biotechnologies }\end{array}$ & $(x 10)$ & & & & \\
\hline $\begin{array}{l}\text { Intervalle de génération } \\
\text { mère-descendant (ans) }\end{array}$ & $4-6$ & $5-6$ & $3-4$ & $4-5$ & $3-4$ \\
\hline
\end{tabular}

* Source : UNCEIA, chiffres de 2010.

b) Mâles

\begin{tabular}{|l|c|c|c|c|c|}
\hline & $\begin{array}{c}\text { Bovins } \\
\text { laitiers }\end{array}$ & $\begin{array}{c}\text { Bovins } \\
\text { allaitants }\end{array}$ & $\begin{array}{c}\text { Ovins } \\
\text { laitiers }\end{array}$ & $\begin{array}{c}\text { Ovins } \\
\text { allaitants }\end{array}$ & Chèvres \\
\hline Semence congelable & oui & oui & (non) & (non) & oui \\
\hline Nb. doses d'IA par an & $\times 10^{4}$ & $\times 10^{4}$ & $\times 100$ & $\times 100$ & $\times 10^{3}$ \\
\hline Nb. total descendants (MN) & $\times 10$ & $\times 10$ & $\times 10$ & $\times 10$ & $\times 10$ \\
\hline Nb. total descendants (IA) & $\times 10^{5}$ max. & $\times 10^{3}$ & $\times 100$ & $\times 100$ & $\times 10^{3}$ \\
\hline $\begin{array}{l}\text { Intervalle de génération père- } \\
\text { descendant (ans) }\end{array}$ & $7-9$ & $5-7$ & 4 & $3-4$ & $3-4$ \\
\hline Utilisation de semence sexée & oui & oui & non & non & non \\
\hline
\end{tabular}

toutefois d'importantes différences entre races, notamment chez les ovins. Finalement, la première saillie ou insémination a lieu au plus tôt à l'âge de 7 mois chez les petits ruminants et entre 15 et 25 mois chez les bovins, dont le temps pour atteindre l'âge adulte est beaucoup plus long, en particulier chez les races allaitantes. Le rythme auquel les femelles de ruminants se reproduisent naturellement est plutôt faible (1 à 1,5 mise bas par an) : il dépend de la durée de la gestation (5 mois chez les petits ruminants, 9 mois chez les bovins) mais aussi de la durée d'allaitement (ovins, bovins allaitants) et parfois de la saison (ovins, caprins). En définitive, l'intervalle de génération entre une femelle et sa descendance s'étend de 3 à 6 ans selon l'espèce, alors que ce même intervalle est de 2-3 ans chez la truie et de seulement 1 an chez la poule ou la lapine.

A cette relative lenteur de la reproduction s'ajoute une faible prolificité des ruminants, en particulier des vaches. Couplés à une carrière plus ou moins longue (plus longue en général pour les femelles allaitantes que les laitières), ces paramètres biologiques conduisent à des nombres totaux de descendants dans la carrière d'une femelle, faibles chez les ovins et caprins (de 6 à 12 descendants), voire très faibles chez les bovins (de 1 à 8 descendants), comparés aux porcins (60-70 descendants en moyenne par truie) ou aux volailles (130-140 descendants en moyenne par poule).

L'induction et la synchronisation des chaleurs, réalisées essentiellement au moyen d'hormones, sont utilisées chez les ovins et les caprins pour contourner les contraintes de saisonnalité et, dans ces mêmes espèces et chez les bovins allaitants, pour mettre en œuvre l'insémination artificielle. Cependant, le coût de ces traitements, la multiplication des interventions sur les animaux, et l'image négative de l'utilisation des hormones en élevage tendent à limiter l'utilisation de ces traitements d'induction des chaleurs, notamment en élevage allaitant. En outre, ces traitements n'augmentent que modérément ou pas du tout le nombre de descendants par femelle. En revanche, les traitements de superovulation (augmentation du nombre d'ovulations par des injections de FSH et LH) associés à certaines biotechnologies de l'embryon mises au point chez les bovins entre les années 70 
(tranplantation et congélation embryonnaires) et les années 90 (ovum pickup/fécondation in vitro ou OPU/FIV) permettent aujourd'hui d'obtenir plusieurs dizaines de descendants par reproductrice et par an et de diminuer l'intervalle de générations mère-descendant. En France, ces biotechnologies, chères et techniquement lourdes, sont essentiellement appliquées aux mères à taureaux d'insémination, principalement dans les races laitières (mais pas exclusivement).

Chez les ruminants mâles, le nombre total de descendants par individu, de quelques dizaines par la monte naturelle dans les trois espèces, est démultiplié par le biais de l'insémination artificielle (cf. tableau 4b). Cette efficacité de l'insémination pour la diffusion des mâles est particulièrement impressionnante chez les bovins du fait d'une assez forte concentration de la semence en spermatozoïdes, de la bonne congélabilité de cette semence, et de la possibilité de déposer la dose inséminante dans l'utérus de la vache (ce qui permet de diminuer le nombre de spermatozoïdes par mie particulière du col utérin nécessite une insémination intra-cervicale (chèvre) ou intra-vaginale (brebis): un nombre plus élevé de spermatozoïdes par dose est nécessaire, ce qui limite le pouvoir de diffusion des mâles. Chez les ovins, la mauvaise congélabilité de la semence limite encore plus le pouvoir de diffusion des mâles, qui sont utilisés presque exclusivement en semence fraîche. Quoi qu'il en soit, dans les trois espèces, l'insémination est une biotechnologie permettant de diffuser le progrès génétique, par la voie mâle, de manière bien plus efficace que les biotechnologies utilisamination offre des garanties sanitaires aux éleveurs et permet de multiples croisements au sein d'un même élevage. Mais l'obligation de détecter les chaleurs, ou à défaut de les induire par traitement hormonal, et la nécessaire condose). Chez les ovins et caprins, l'anatobles sur la voie femelle. En outre, l'insé-

Photo 2. Bouc de race Poitevine.

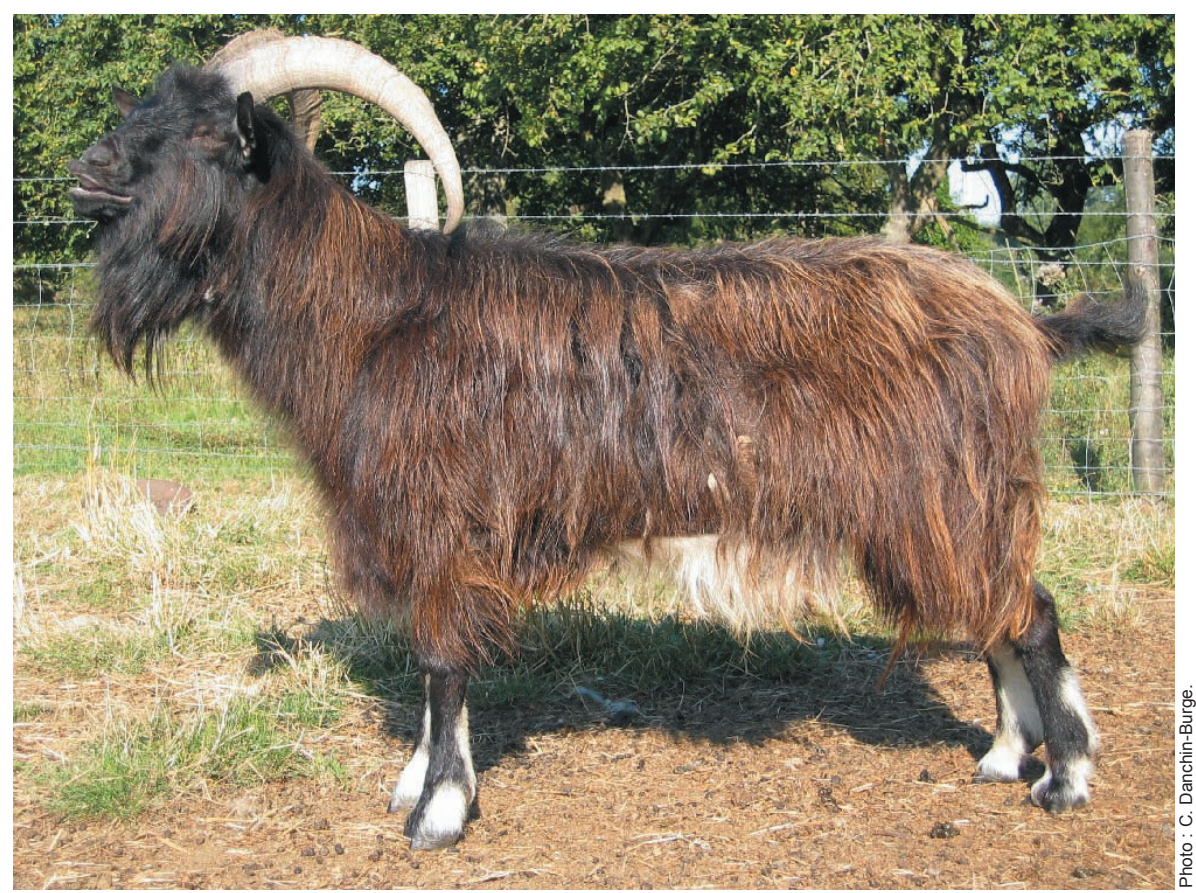

tention des femelles à inséminer, n'ont pas facilité le développement de l'insémination dans certains systèmes d'élevage, notamment les élevages allaitants.

\section{3 / La diversité des milieux exploités, des systèmes d'éle- vage et des produits : conséquences pour le choix des races et des objectifs de sélection}

\section{1 / Diversité de l'élevage des ruminants}

Dans un pays comme la France, l'élevage des ruminants est pratiqué sur des territoires variés et avec une gamme diversifiée de systèmes d'élevage, depuis des systèmes très intensifs avec
Tableau 5. Nombre de races et importance relative de celles aux plus grands effectifs selon l'espèce et la filière.

\begin{tabular}{|l|c|c|c|c|c|}
\hline & $\begin{array}{c}\text { Bovins } \\
\text { laitiers }\end{array}$ & $\begin{array}{c}\text { Bovins } \\
\text { allaitants }\end{array}$ & $\begin{array}{c}\text { Ovins } \\
\text { laitiers }\end{array}$ & $\begin{array}{c}\text { Ovins } \\
\text { allaitants }\end{array}$ & Chèvres \\
\hline $\begin{array}{l}\text { Nb. total de races } \\
\text { reconnues }\end{array}$ & 20 & 26 & 6 & 51 & 8 \\
\hline $\begin{array}{l}\text { Dont races avec plus } \\
\text { de 100 000 têtes }\end{array}$ & 3 & 5 & 3 & 12 & 2 \\
\hline $\begin{array}{l}\text { Part du cheptel national } \\
\text { représenté par les races } \\
\text { à plus de 100 000 têtes }\end{array}$ & $96 \%$ & $96 \%$ & $90 \%$ & $65 \%$ & $99 \%$ \\
\hline
\end{tabular}

Source : Ministère de l'Agriculture. recours important aux cultures fourragères et aux aliments concentrés, fréquents en élevage laitier dans les trois espèces, jusqu'à des systèmes très extensifs valorisant essentiellement des herbages et des parcours plus ou moins arborés. Cette diversité est évidemment plus grande encore si l'on élargit l'horizon géographique...

Les produits de cet élevage sont euxmêmes diversifiés et leurs marchés sont très segmentés : à côté d'une demande persistante des consommateurs pour des produits «de masse» à bas prix, toute une gamme de produits «typiques», «de qualité», «de niche», voire «de luxe», s'est développée et, avec eux, des filières différenciées et tout un dispositif de signes officiels censés garantir aux consommateurs les caractéristiques qu'ils recherchent dans ces produits : les signes français Appellation d'Origine Contrôlée (AOC) et Label Rouge, et les signes européens Appellation d'Origine Protégée (AOP), Indication Géographique Protégée (IGP), Agriculture Biologique (AB en France), et Spécialité Traditionnelle Garantie (STG). La France n'est certes pas le seul pays d'Europe à présenter une grande variété de produits mais c'est un de ceux qui a cultivé le plus cette diversité.

En partie du fait de cette diversité de situations, il y a en France une grande diversité de races d'une espèce donnée pour une production donnée (tableau 5). Certes, les effectifs de ces races sont inégaux et un petit nombre de races, spécialisées, dominent nettement (sauf 
en ovins allaitants); cette diversité est cependant plus élevée que dans la plupart des autres pays européens (voir par exemple, FAO 2011).

\section{2 / La définition des objectifs de sélection}

La liste des caractères que l'on souhaite améliorer ainsi que la hiérarchie que l'on fait entre eux constituent les objectifs de sélection. Ils sont établis pour chaque race séparément, du fait notamment de la diversité des situations évoquée plus haut. Cela nécessite de se projeter dans l'avenir car le délai entre une décision de sélection et ses répercussions à grande échelle, c'est-à-dire lors de l'expression des caractères chez les descendants des reproducteurs sélectionnés, se compte en années (cf. les valeurs des intervalles de génération, tableau 4). La notion d'animal amélioré variant d'un acteur à l'autre des filières, le choix des objectifs de sélection relève toujours de l'art du compromis. En France, ce compromis est recherché (et en général trouvé) au sein des Organismes de Sélection (OS), dont la définition des objectifs de sélection est une des prérogatives. Ces OS regroupent quatre types d'acteurs directement concernés par la race en question : des éleveurs à titre individuel, des entreprises de sélection, des organismes des filières de production (par ex., groupement de producteurs, syndicat de défense d'AOP...) et des organismes des territoires correspondants (parc naturel, syndicat d'aménagement...).

Un regard sur les dernières décennies permet de montrer que les objectifs de sélection évoluent dans le temps (pour les bovins laitiers, voir Verrier et al
2010). Schématiquement, l'amélioration de la productivité a constitué un objectif majeur dans les années 60 à 70 : elle a concerné la quantité de lait par lactation, la vitesse de croissance, la prolificité chez les petits ruminants, etc. Dès les années 70, ont été prises en compte les caractéristiques des produits : les taux de matière protéique et de matière grasse du lait, les caractéristiques des carcasses, etc. A partir des années 80/90, les caractères dits fonctionnels, c'est-à-dire qui facilitent l'élevage et la reproduction des animaux, ont été progressivement intégrés jusqu'à représenter ensemble la moitié environ des objectifs et des efforts de sélection : il s'agit de la fertilité, la longévité, la résistance à certaines maladies, etc. Dans les années à venir, on peut pronostiquer la prise en compte dans les objectifs de nouvelles caractéristiques des produits, comme celles issues de l'analyse fine de la composition du lait, par exemple. La recherche d'animaux adaptés à leurs conditions d'élevage, robustes et autonomes, sera également poursuivie, par exemple à travers l'accroissement du poids des caractères fonctionnels dans les index de synthèse des bovins laitiers.

\section{4 / L'évaluation génétique des animaux et l'informa- tion requise}

\section{1 / Les informations disponi- bles et leur circuit}

L'identification est une pratique, ancienne chez les bovins (encore plus chez les chevaux), plus récente chez les petits ruminants. Elle a été généralisée

Photo 3. Veau Prim'Holstein avec ses boucles d'identité : l'identification est un préalable à de nombreuses actions zootechniques, notamment l'amélioration génétique.

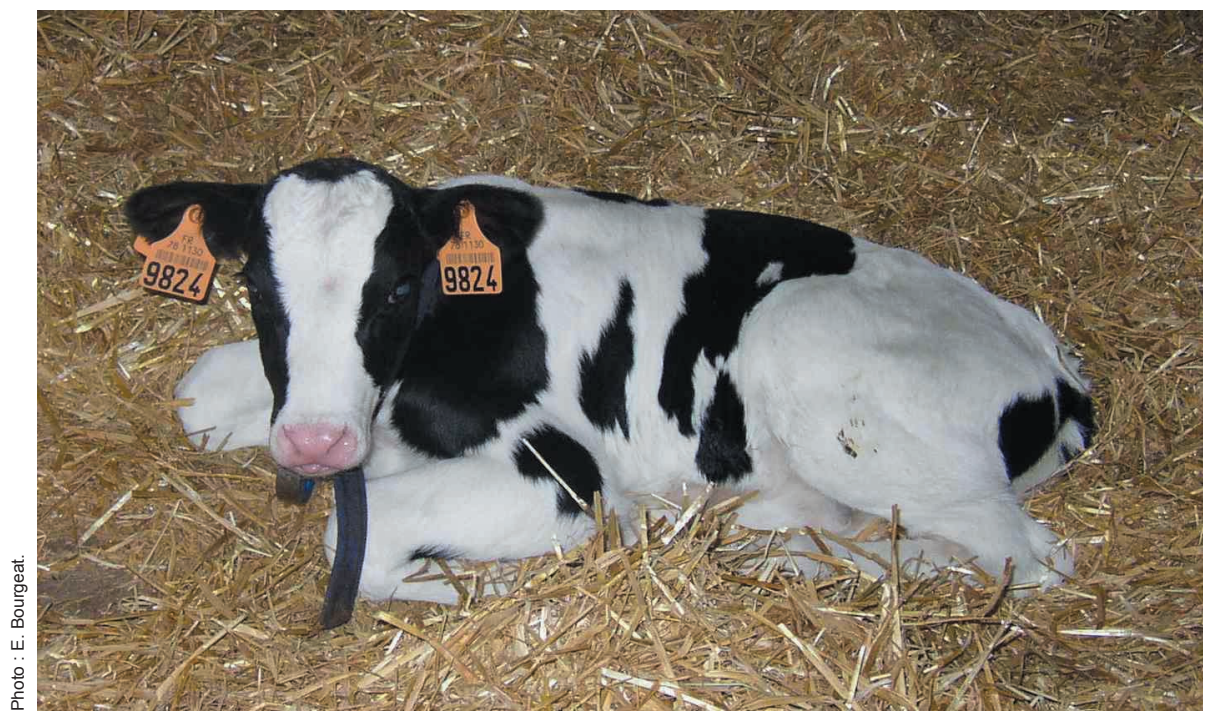

pour d'autres motifs que l'amélioration génétique : traçabilité, prophylaxie, attribution de primes, etc. Le marquage des animaux est obligatoire dès leur naissance et les boucles actuellement en vigueur sont fiables. L'identification électronique est en cours de développement chez les bovins et elle est obligatoire pour tous les petits ruminants nés depuis le $1^{\text {er }}$ juillet 2010.

Le recueil de l'état civil est lui aussi pratiqué depuis longtemps. Reposant sur les déclarations des éleveurs, il s'accompagne en premier lieu du contrôle de la cohérence entre dates déclarées de mise à la reproduction et de naissance. Ce dispositif est complété par des contrôles de filiation à l'aide de marqueurs moléculaires, au titre de sondage chez les bovins, et obligatoires dans certains cas comme les futurs reproducteurs d'insémination artificielle (de l'ordre de 50000 contrôles de filiation effectués chaque année en France pour les bovins).

Chez les ruminants, le contrôle des performances en ferme est apparu dès le début du vingtième siècle et s'est surtout développé dans sa seconde moitié. Aujourd'hui la France se situe dans le peloton de tête à l'échelle mondiale pour l'ampleur de cette activité. En 2010, dans les filières laitières, environ $67 \%$ des vaches, $58 \%$ des brebis et $44 \%$ des chèvres sont soumises au contrôle des performances; dans les filières allaitantes, les taux de contrôles sont plus faibles, avec environ $22 \%$ des vaches et $7 \%$ des brebis (FGE 2011).

Les marqueurs moléculaires, enfin, sont disponibles en routine depuis la fin des années 80 (la première application, le génotypage des taureaux laitiers pour le gène de la K-caséine, date de 1988). Après avoir longtemps employé des marqueurs microsatellites, très polymorphes (de l'ordre de 6-7 allèles par marqueur) mais relativement peu nombreux (plusieurs dizaines de milliers par génome chez les ruminants), aujourd'hui, on utilise (ou cherche à utiliser) des marqueurs SNP (Single Nucleotide Polymorphism), peu polymorphes (2 allèles par marqueur) mais extrêmement nombreux (quelques millions par génome). Des puces à SNP, permettant de génotyper un animal pour des dizaines de milliers de marqueurs à la fois, sont d'ores et déjà disponibles chez les bovins et les ovins et sur le point de l'être chez les caprins.

Les différentes informations collectées sur le terrain (identité, état civil, performances) ou obtenues en laboratoire à partir de prélèvements biologiques effectués sur le terrain (génotype à des marqueurs) sont centralisées, via un réseau de centres régionaux informa- 
Photo 4. Vaches Aubrac au pâturage.

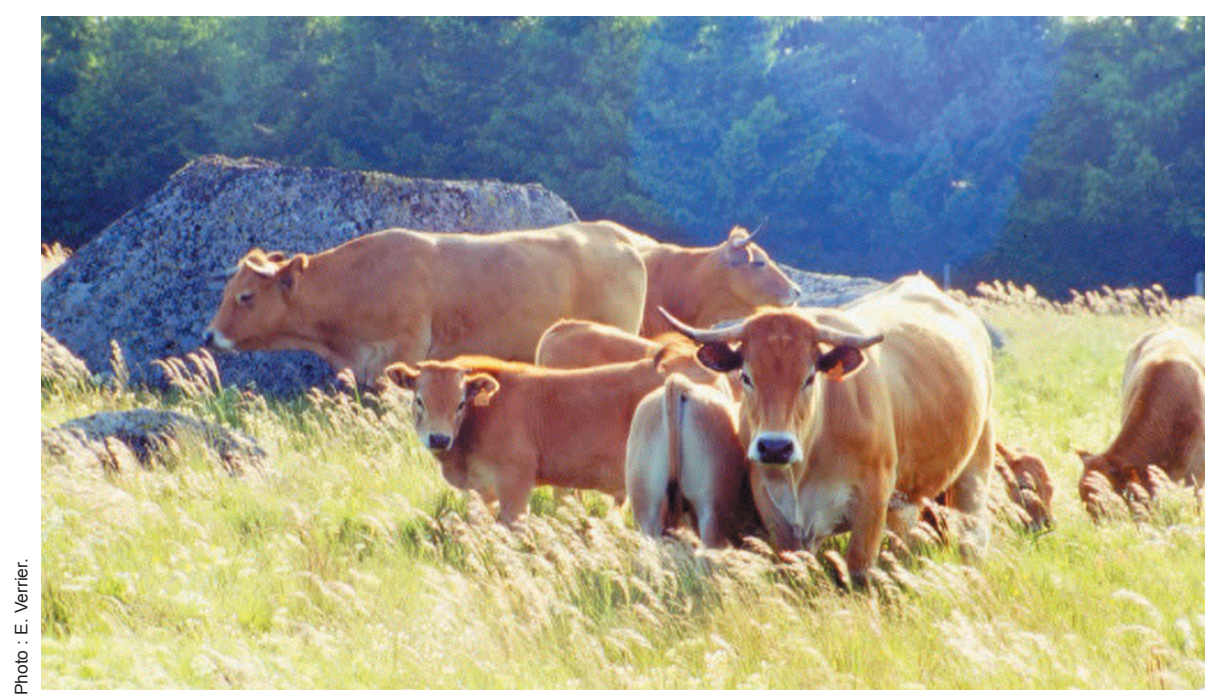

tiques, dans des bases de données du type «Système National d'Information Génétique» dédiées à chaque espèce et/ou filière. Ces bases sont maintenues au Centre de Traitement de l'Information Génétique (CTIG), à Jouy-en-Josas. L'Etat et l'INRA ont la responsabilité de l'échelon central, l'interprofession France Génétique Elevage (FGE) contribuant à la gestion et à la maintenance des systèmes d'information, depuis les élevages jusqu'aux bases nationales et vice-versa.

\section{2 / L'évaluation génétique}

L'évaluation génétique consiste à estimer (en toute rigueur prédire) la valeur génétique d'un animal. Cette procédure est très généralement désignée par le terme d'indexation, les index de valeur génétique représentant la valeur génétique estimée des animaux et servant à classer les candidats à la sélection selon leur mérite (d'où le terme d'index).

Comme évoqué plus haut, chez les ruminants, les animaux sont répartis dans un grand nombre d'élevages. Ils ont des carrières plus ou moins longues (tableau 4) et réalisent de multiples performances, lors d'années différentes. Il résulte de ces deux faits de grandes différences de conditions d'environnement dans lesquelles les performances ont été réalisées, à la fois dans l'espace et dans le temps. Afin de dissocier au mieux les valeurs génétiques et les effets d'environnement, il est alors nécessaire d'avoir recours à des méthodes statistiques élaborées, dans le cadre de la méthodologie BLUP (Best Linear Unbiased Predictor).

Mission régalienne de l'INRA dans le cas des ruminants en France, la mise au point des méthodes d'évaluation génétique constitue un champ actif de recherche. Parmi les innovations majeu- res dans ce domaine, citons $i$ ) la prise en compte de l'hétérogénéité des variances (Robert-Granié et al 1999), ii) l'évaluation assistée par des marqueurs de QTL (Quantitative Trait Loci) donnant accès, chez les bovins laitiers depuis 2001, à la Sélection Assistée par Marqueurs (SAM) et iii) l'évaluation à partir de données SNP obtenues «en masse» (cf. plus haut) donnant accès, chez les bovins laitiers depuis 2009 et à venir dans les autres espèces, à la sélection née à l'approche QTL sus-évoquée (cf. Mulsant et al 2011).

En définitive, en matière de recueil de l'information zootechnique et de son utilisation pour l'évaluation génétique, les ruminants possèdent de très solides atouts, parmi lesquels une proportion importante d'animaux concernés et donc de larges bases de sélection, des bases de données riches et remontant loin dans le temps, un savoir-faire et une autonomie bien établis en matière d'évaluation génétique, une grande fiabilité des procédures mises en œuvre - procédures qui font l'objet d'un Système de Management de la Qualité (SMQ), l'évaluation génétique faisant l'objet depuis 2006 d'une certification ISO-9001 -, et une organisation impliquant les différents corps de métier dans un cadre bien défini.

\section{5 / Les possibilités de choix des reproducteurs et de création du progrès géné- tique}

\section{1 / Les paramètres du progrès génétique annuel}

C'est le choix raisonné des reproducteurs de renouvellement parmi un génomique qui, en France, est combi- ensemble de candidats à la sélection qui permet de dégager un progrès génétique dans le sens des objectifs définis au préalable (cf. plus haut). Le rythme avec lequel la moyenne des valeurs génétiques pour un caractère va évoluer au cours du temps sous l'effet de la sélection dépend de quatre paramètres :

- la variabilité génétique au sein de la population : plus l'écart-type génétique additif $\left(\sigma_{a}\right)$ du caractère sélectionné est élevé, plus la marge d'évolution attendue est grande ;

- la sévérité du choix : plus la proportion de reproducteurs sélectionnés parmi l'ensemble des candidats $(p)$ est faible, plus l'intensité de sélection $(i)$ est forte et plus le gain génétique est élevé ;

- la précision de la sélection : c'est la corrélation entre le critère de sélection (en général la valeur génétique estimée) et la valeur génétique vraie des animaux, égale à la racine carrée du Coefficient de Détermination $(C D)$ des index de valeur génétique lorsque ce sont eux qui sont employés comme critères de sélection (ce qui est recommandé...) ;

- l'intervalle de génération $(T)$ : c'est la durée qui sépare la naissance des reproducteurs de celle de leurs descendants qui deviendront à leur tour reproducteurs.

En définitive, l'espérance du progrès génétique annuel $\left(\Delta G_{a}\right)$ s'exprime comme suit :

$$
\mathrm{E}(\Delta G a)=i \times C D^{1 / 2} \times \sigma_{a} / T
$$

\section{2 / Caractéristiques des rumi- nants vis-à-vis de la création du progrès génétique}

L'espérance du progrès génétique annuel dépend, d'une part, de certaines caractéristiques biologiques de l'espèce considérée (rythme de reproduction, fécondité des reproducteurs...) et, d'autre part, des méthodes de sélection appliquées. La situation des ruminants par rapport aux paramètres du progrès génétique annuel appelle les commentaires suivants (cf. également tableau 4) :

- La lenteur du renouvellement des générations, notamment chez les bovins, constitue en quelque sorte une «force d'inertie», accentuée par la pratique de l'épreuve de la descendance des mâles. Jusqu'en 2006, cette épreuve était généralisée dans le cas des mâles destinés à l'insémination, compte tenu de leur large diffusion potentielle et, donc, de la nécessité de les évaluer avec un $C D$ élevé (ce qui constitue une garantie apportée à l'usager). Aujourd'hui, deux facteurs permettent d'envisager de 
se dispenser de l'épreuve de la descendance des mâles, et donc de raccourcir sensiblement les intervalles de génération. D'une part, dans le cadre de la LOA de 2006 et de l'harmonisation avec la réglementation européenne, les conditions zootechniques d'accès à l'insémination ont été assouplies. D'autre part, la sélection génomique, mise en œuvre depuis 2009 en bovins laitiers, permet d'atteindre des $C D$ corrects, compatibles avec la réglementation européenne $(C D$ minimal de 0,5 pour les bovins laitiers, par exemple), quelle que soit l'héritabilité du caractère ;

- L'importance des bases de sélection (animaux contrôlés) est favorable à l'intensité de sélection mais il existe des différences d'un sexe à l'autre. Les femelles de ruminants étant peu fécondes, il y a peu de marge de tri sur la voie mère-fille, seule voie qui incombe aux éleveurs individuels. L'accès au génotypage des femelles et, chez les bovins, l'utilisation de semence sexée (depuis 2000), devraient permettre d'accroître l'efficacité de cette voie. Les mâles d'insémination ayant une capacité de diffusion importante (petits ruminants) à très large (bovins), ils peuvent être peu nombreux, ce qui permet de très fortes intensités de sélection dans le choix de leur père (voie père-fils) et de leur mère (voie mère-fils). Compte tenu des différences entre espèces et filières quant à la proportion de femelles inséminées (cf. tableau 4a) et du nombre de doses qu'il est possible de produire par reproducteur (cf. tableau 4b), cet impact favorable est très marqué chez les bovins lai- tiers, dans une moindre mesure chez les ovins laitiers et les caprins, et modéré chez les bovins et ovins allaitants ;

- Le tri (très) sévère des parents des mâles d'insémination engendre des «goulets d'étranglement» au sein des populations : certains reproducteurs apparaissent très fréquemment dans les pedigree et ont donc une contribution génétique très élevée à l'ensemble des animaux actuels. Cela entraîne une réduction de la variabilité génétique qui réduit, voire peut compromettre, les possibilités d'évolution future. Ces phénomènes touchent particulièrement les bovins laitiers (voir, par exemple, Danchin-Burge et al 2011) et plusieurs dispositions ont été prises pour en limiter l'ampleur : communication, dans les catalogues de taureaux d'insémination, du coefficient de parenté entre un taureau donné et l'ensemble des femelles de la race ; mise en place d'un observatoire de la variabilité génétique (projet CASDAR «VARUME» piloté par l'Institut de l'Elevage); mise en œuvre de procédures optimisées de sélection et d'utilisation des reproducteurs (programme «VARGEN», cf. Colleau et al 2004). Par ailleurs, la sélection génomique fournissant des $C D$ légèrement plus faibles que l'épreuve de la descendance pour les caractères laitiers (Collectif 2010), son développement devrait conduire à des choix plus diversifiés de taureaux et à la fin du «star system». Des études de simulation montrent en outre que, sous réserve de l'utilisation équilibrée d'un grand nombre de reproducteurs, la sélection géno-

Photo 5. Béliers de race Manech Tête Noire, l'une des trois races ovines laitières des Pyrénées.

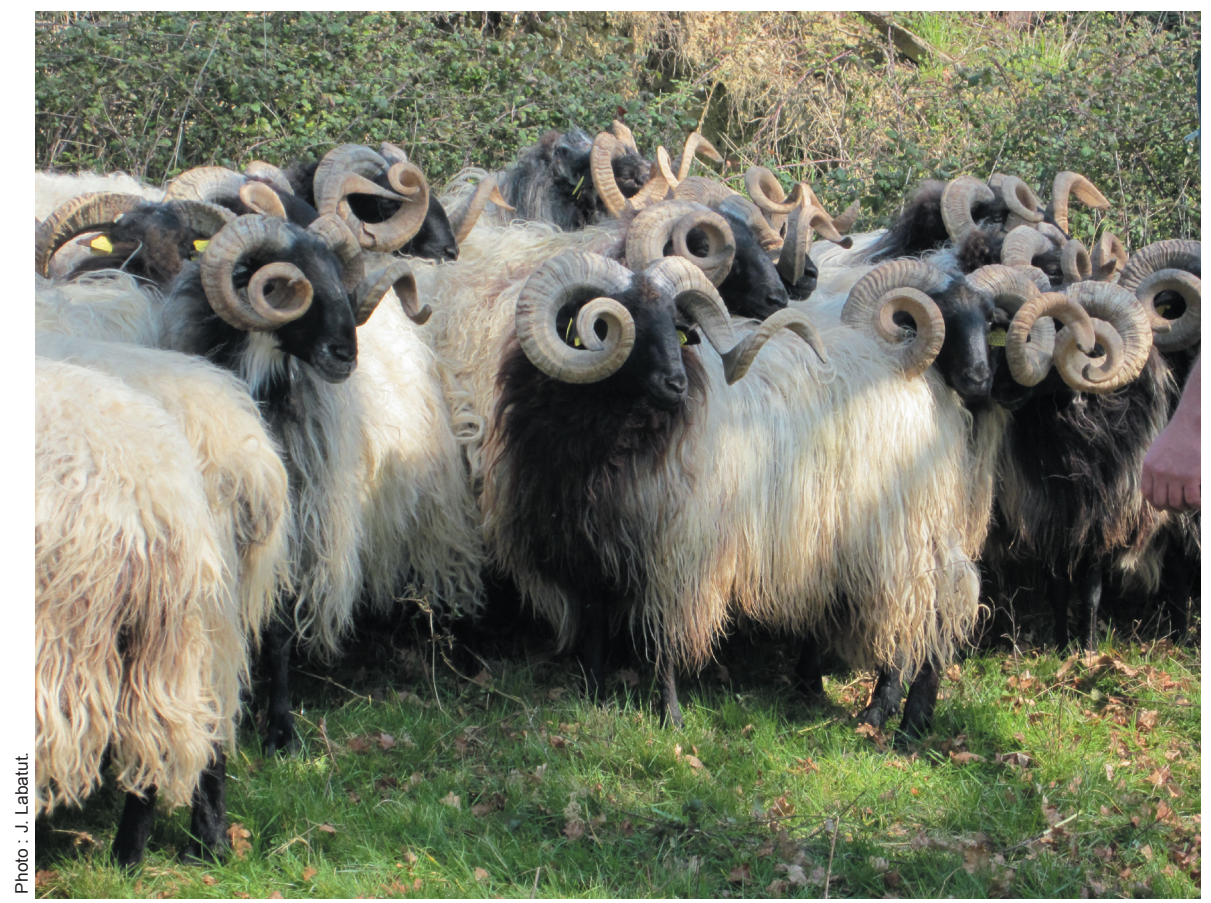

mique peut conduire à un rythme modéré d'accroissement de la consanguinité malgré le raccourcissement de l'intervalle de génération induit par cette méthode (Colleau et al 2009);

- Un autre apport majeur de la sélection génomique chez les ruminants est l'accroissement de l'efficacité de la sélection sur des caractères très peu héritables (la fertilité, par exemple) grâce à l'obtention de $C D$ corrects, tant pour les mâles que pour les femelles, substantiellement plus élevés qu'à l'issue de l'épreuve de la descendance des mâles pour ces caractères-là.

\section{6 / Les possibilités d'utilisa- tion des reproducteurs et de diffusion du progrès géné- tique}

\section{1 / Utilisation au sein de la population}

Au sein d'une population donnée, l'utilisation des reproducteurs recouvre deux réalités. D'une part, il est nécessaire de recycler le progrès génétique au sein de la base de sélection, c'est-à-dire faire en sorte que les prochaines générations de candidats bénéficient de ce progrès. En pratique, il s'agit de cumuler les progrès génétiques de génération en génération, en procréant les futurs candidats à la sélection à partir d'accouplements raisonnés entre les meilleurs reproducteurs disponibles à un moment donné. D'autre part, il est nécessaire de diffuser le progrès génétique en dehors de la base de sélection afin d'en faire bénéficier l'ensemble des éleveurs (ce qui permet également de trouver des ressources financières pour couvrir une partie du coût des programmes).

Compte tenu de la faible fécondité des femelles, même avec le recours aux biotechnologies (tableau 4a), le recyclage du progrès génétique, et plus encore sa diffusion, reposent principalement sur la voie mâle. Là encore, la maîtrise de l'insémination constitue un atout décisif. En ce qui concerne le recyclage du progrès génétique, l'insémination permet de réaliser exactement les accouplements raisonnés que l'on souhaite, indépendamment de la localisation des animaux. En matière de diffusion, il convient de nettement distinguer les bovins des ovins, les taureaux ayant un très large potentiel de diffusion et les béliers un potentiel fort modéré, les caprins étant dans une position intermédiaire (tableau 4b). Ainsi, les taureaux d'insémination sont utilisés pour procréer des filles de renouvellement dans les élevages. Chez les petits ruminants, en revanche, c'est essentiellement 
via les fils de monte naturelle que les mâles d'insémination diffusent leurs gènes hors des élevages contrôlés.

\section{2 / Race pure ou croisement}

En ce qui concerne l'utilisation des reproducteurs, il convient de distinguer également deux modes d'élevage : l'élevage dit «en race pure» (inutile de préciser qu'il n'y a pas de jugement de valeur dans cette expression), qui consiste à faire se reproduire entre eux des géniteurs issus de la même population, et le croisement, qui consiste à faire se reproduire entre eux des géniteurs issus de populations différentes. L'élevage en race pure a le mérite de la simplicité et permet de renouveler les populations, ce qui est toujours nécessaire même quand une race est utilisée en croisement. Chez les ruminants, l'usage du croisement est variable selon la filière.

Dans les filières laitières, le croisement est employé à deux fins. D'une part, essentiellement chez les bovins, le but est d'intégrer des allèles présents dans d'autres races, soit à dose «homéopathique» comme lors du croisement passager des races Montbéliarde et Abondance avec la Holstein rouge, soit à dose massive comme lors de l'absorption ou la quasi-absorption des races Frisonne et Brune par leurs homologues Nord-Américaines (pour une évolution des proportions de gènes des différentes races, voir Danchin-Burge et al 2011). Il s'agit là d'une démarche collective, décidée avec plus ou moins de controverses au sein des Organismes de Sélection concernés. D'autre part, le but est d'apporter, via un mâle issu d'une race allaitante spécialisée, une plusvalue bouchère aux jeunes nés de certaines femelles présentes dans le troupeau mais dont on est sûr de ne pas vouloir conserver la descendance. Il s'agit là d'une démarche individuelle, entreprise indépendamment d'un éleveur à l'autre.

Dans les filières allaitantes, le croisement est principalement employé pour tirer parti de la complémentarité entre des races à aptitudes maternelles et/ou de rusticité, d'une part, et des races bouchères spécialisées, d'autre part. En France, lorsqu'il est mis en œuvre, ce type de croisement est le plus souvent organisé sur une seule génération (croisement simple). Des exemples de ce type de croisement à grande échelle sont fournis par les races bovines Salers et Aubrac dont 50 à $60 \%$ des femelles sont croisées avec des taureaux de races bouchères, Charolais pour l'essentiel, ou la race ovine Mérinos d'Arles dont environ la moitié des femelles sont croisées avec des béliers de races bouchères, le Berrichon du Cher principalement. Chez les ruminants, des plans de croisement sur deux générations (croisement à double étage) sont mis en œuvre beaucoup plus rarement: le seul exemple à grande échelle est celui de la race ovine Causses du Lot, les mâles utilisés étant successivement de race Ile-de-France et Berrichon du Cher. Dans tous les cas, le renouvellement de la race maternelle ou rustique repose sur la moitié (ou moins) des femelles, ce qui, compte tenu de leur faible fécondité, suppose des carrières longues et régulières afin de procréer le nombre requis de filles. Signalons enfin que l'élevage ovin extensif en Limousin fait intervenir des croisements de type rotatif, qui n'ont toutefois pas le côté systématique des plans évoqués cidesssus.

Le croisement est donc utilisé chez les ruminants mais, en France (comme dans bon nombre de pays européens), l'élevage en race pure est encore prépondérant. Ceci contraste avec la situation des volailles et des porcs, où l'élevage et les filières sont très fortement structurés autour de plans de croisement à double étage (Coudurier 2011). En effet, l'usage du croisement dépend de certaines caractéristiques de l'espèce concernée (mise en place plus aisée dans des espèces dont les femelles sont très fécondes), des possibilités d'organisation et de segmentation des métiers au sein d'une filière et de l'importance accordée à l'image du reproducteur, mâle notamment. La faible fécondité des femelles de ruminants et la dispersion des élevages ne laissent pas toute la latitude voulue pour organiser une filière autour de plans de croisement (en dehors des cas évoqués plus haut). Par ailleurs, très nettement pour les bovins, dans une moindre mesure pour les petits ruminants, les éleveurs sont encore très attachés à utiliser des reproducteurs mâles de race pure.

\section{Conclusion}

Sans doute démarrée de façon empirique dès les débuts de la domestication, la sélection des ruminants s'est développée dans le cadre de programmes intégrés depuis la seconde moitié du 20 ème siècle. Compte tenu du coût d'entretien des animaux et des pas de temps dans ces espèces, ces programmes sont par essence collectifs. Leur efficacité est à l'évidence pénalisée par la faible fécondité des femelles et la lenteur du renouvellement des générations dans ces espèces. Toutefois, de nombreux éléments, de diverses natures, sont très favorables à la création et à la diffusion de progrès génétiques substantiels : une organisation collective rigoureuse, dans un cadre réglementaire précis ; des objectifs de sélection clairs et, en général, largement partagés par les différents acteurs des filières; des contrôles de performances très développés et de larges bases de sélection; l'accès aux informations génomiques à haute densité ; des circuits bien établis de collecte et de traitement de l'information et un savoir-faire reconnu en matière d'évaluation génétique ; pour les bovins, une très bonne maîtrise de l'insémination artificielle, traduite par de très bons résultats techniques.

La sélection génomique, démarrée chez les bovins laitiers et qui va très vite concerner les autres espèces ou filières, ouvre de nouvelles perspectives, tout particulièrement le raccourcissement des intervalles de génération, la prise en compte d'un nombre accru de caractères et la sélection efficace des caractères très peu héritables (cf. Mulsant et al 2011). Il n'est pas exagéré de dire que cette méthode de sélection révolutionne ou va révolutionner la pratique de l'amélioration génétique : un exemple concret en est, depuis juin 2009, l'autorisation d'utilisation à grande échelle de taureaux laitiers sans descendants connus, évalués uniquement sur la base de leur information génomique. A n'en pas douter, ce saut méthodologique nécessite un suivi attentif des conséquences sur les pratiques et sur l'organisation de l'amélioration génétique, ainsi qu'un accompagnement en matière de formation des différents acteurs de la sélection. 


\section{Références}

AgroParisTech-CSAGAD, Institut de l'Elevage, 2006. Après la Loi d'Orientation Agricole, quelle organisation et quelle réglementation pour la sélection animale ? http://www.agroparistech.fr/svs/genere/ger_ge netique/csagad/loa/loa programme.htm (consultée le 30 août 2011).

Colleau J.J., Moureaux S., Briend M., Béchu J., 2004. A method for the dynamic management of genetic variability in dairy cattle. Genet. Sel. Evol., 36, 373-394.

Colleau J.J., Fritz S., Guillaume F., Baur A., Dupassieux D., Boscher M.Y., Journaux L., Eggen A., Boichard D., 2009. Simulation des potentialités de la sélection génomique chez les bovins laitiers. Renc. Rech. Rum., 16, 419.

Collectif, 2010. Sélection génomique et changements dans l'indexation des bovins laitiers : évolution ou révolution ? Institut de l'Elevage, AgroParisTech-CSAGAD, 27p.

Coudurier B., 2011. Contraintes et opportunités d'organisation de la sélection dans les filières porcine et avicole. In : Numéro spécial, Amélioration génétique. Mulsant P., Bodin L., Coudurier B., Deretz S., Le Roy P., Quillet E., Perez J.M. (Eds). INRA Prod. Anim., 24, 307322.

Danchin-Burge C., Leroy G., Brochard M., Moureaux S., Verrier E., 2011. Evolution of the genetic variability of 8 French dairy cattle breeds assessed by pedigree analysis. J. Anim. Breed. Genet. (sous presse).

Denis B., 1982. La notion de race : point de vue d'un ethnozootechnicien. Ethnozootechnie 29, 61-67.

FAO, 2011. Domestic Animals Diversity Information System (DAD-IS). http://dad. fao.org (consultée le 30 août 2011).

FGE (France Génétique Elevage), 2011. Dispositif génétique : chiffres clés ruminant 2010. http://www.inst-elevage.asso.fr/IMG/ pdf CR 1170003-chiffres cles rumin FGE $201 \overline{0}$.pdf (consultée le 30 août 2011 ).
Lerner M.I., Donald H.P., 1966. Modern developments in animal breeding. Academic Press, London and New York, 294p.

Mulsant P., Bodin L., Coudurier B., Deretz S., Le Roy P., Quillet E., Perez J.M., 2011. Amélioration génétique. Numéro spécial, INRA Prod. Anim., 24, 128p.

Robert-Granié C., Bonaïti B., Boichard D., Barbat A., 1999. Accounting for variance heterogeneity in French dairy cattle genetic evaluation. Livest. Prod. Sci,, 60, 343-357.

Verrier E., 2011. L'amélioration génétique : une activité humaine organisée, au service de l'adaptation des populations animales. Journ. Rech. Equine, 37, 85-92.

Verrier E., Le Mézec P., Boichard D., Mattalia S., 2010. Evolution des objectifs et des méthodes de sélection des bovins laitiers. Bull. Acad. Vét. Fr., 163, 73-78.

\section{Résumé}

Après un aperçu des cheptels de ruminants élevés en France et dans l’Union Européenne, les caractéristiques des espèces qui influent sur les possibilités de mettre en œuvre des programmes d'amélioration génétique et sur leur efficacité sont mentionnées. L'accent est mis sur les conséquences des paramètres de la reproduction, sur la nécessité d'une organisation rigoureuse et sur la diversité des situations rencontrées, entre espèces, races, territoires et filières. Les perspectives offertes par le développement en cours de la sélection génomique sont brièvement évoquées.

\section{Abstract}

\section{Opportunities and constraints for ruminants breeding}

After an overview of ruminant stocks in France and the European Union, The characteristics of the species acting on the possibilities to develop breeding programs and their efficiency are shown. Emphasis is put on the impact of the efficiency of reproduction, the need for a strict organization and the diversity of cases among species, breeds, territories and market chains. The perspectives opened by the ongoing development of genomic selection are briefly evoked.

VERRIER E., SAINT-DIZIER M., 2011. Contraintes et opportunités pour l'amélioration génétique des ruminants. In : Numéro spécial, Amélioration génétique. Mulsant P., Bodin L., Coudurier B., Deretz S., Le Roy P., Quillet E., Perez J.M. (Eds). INRA Prod. Anim., 24, 297-306. 